\title{
A victory for the Halsteadians?
}

\author{
Nahush A. Mokadam, MD
}

\author{
From the Division of Cardiothoracic Surgery, University of Washington, Seattle, Wash. \\ Funding Sources: None \\ Disclosures: Dr Mokadam serves as a consultant to HeartWare, Thoratec, SynCardia, and St Jude Medical. Author \\ has nothing to disclose with regard to commercial support. \\ Received for publication Aug 31, 2015; accepted for publication Sept 1, 2015; available ahead of print Oct 7, \\ 2015 . \\ Address for reprints: Nahush A. Mokadam, MD, Division of Cardiothoracic Surgery, University of Washington, \\ 1959 NE Pacific, Box 356310, Seattle, WA 98195-6310 (E-mail: mokadamn@uw.edu). \\ J Thorac Cardiovasc Surg 2015;150:1438-9 \\ 0022-5223/\$36.00 \\ Copyright $@ 2015$ by The American Association for Thoracic Surgery \\ http://dx.doi.org/10.1016/j.jtcvs.2015.09.001
}

"I don't want any students or residents operating on me" is a statement heard time and again in teaching institutions. Concerns related to duty hours and supervision have created a perception that teaching hospitals may expose patients to increased risk. ${ }^{1,2}$ Although most teaching centers are able to address these concerns directly with patients, the concept of poorly supervised resident physicians remains common in the media and entertainment industry.

In this issue of the Journal, Almassi and colleagues ${ }^{3}$ describe a retrospective subgroup analysis of a prospective, randomized trial examining results of on- versus off-pump coronary artery bypass grafting. It specifically examines graft patency comparing anastomoses performed by resident physicians with those performed by attending surgeons. The authors demonstrate that outcomes were not affected by method of revascularization or who performed the anastomosis.

This study is incredibly encouraging for resident physician education. It appears to show that surgical educators are succeeding! With appropriate supervision, patient outcomes are not compromised even when a surgeonin-training conducts the operation. This has positive implications for patients, hospital administrators, payors, and regulatory bodies. Further, these findings give significant weight to insistence by the Accreditation Council on Graduate Medical Education and Residency Review Council of resident physician involvement in all aspects of training, including procedures regarded as novel, advanced, or more complex.

On the other hand, there are notable concerns about the findings. First, although a wealth of data were collected, there is no information explaining what determined who performed which anastomosis. As all surgical educators understand, the potential bias can be substantial. Did the attending physicians perform the "worst" and leave the "easier" anastomoses to the resident physicians? Although there was an attempt at describing target quality in terms of

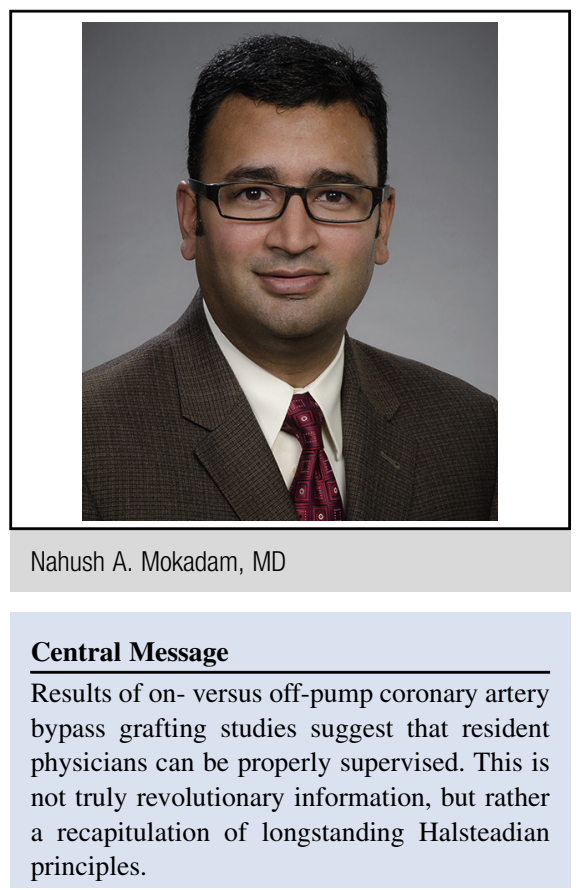

See Article page 1428

See Editorial page 1393.

size and quality, these measures are coarse. There is a further limitation in the definitions of "resident performed" versus "attending performed." Does "performed" mean that $100 \%$ of the anastomosis was completed by a single individual? Does "performed" mean $75 \%$ ? Or $51 \%$ ? Is there consistency or accuracy in this definition? Did the attending move the table under the resident or did the resident set it up?

In the current age, allowing resident physicians real operative experiences while maintaining outstanding outcomes is paramount. In the absence of a randomized trial (which would be impossible to conduct) or without adjudication of the resident physician's role in each and every case (also impossible to conduct), the statement that "residents can be trained in new, complex surgical techniques while maintaining good patient surgical outcomes" 3 is difficult if not impossible to prove.

In their closing remarks, the authors include, "...the validity of the conclusion that residents are able to safely and effectively perform on- and off-pump CABG with attending supervision remains unmitigated." 3 I believe this is an overstatement. This is not truly revolutionary 
information, but rather a recapitulation of longstanding Halsteadian principles.

\section{References}

1. Itani KM, DePalma GH, Schifftner T, Sanders KM, Chang BK, Henderson WG, et al. Surgical resident supervision in the operating room and outcomes of care in Veterans Affairs hospitals. Am J Surg. 2005;190:725-31.
2. Berg DB, Engel AM, Saba A, Hatton EK. Differences in public belief and reality in the care of operative patients in a teaching hospital. J Surg Educ. 2011;68:10-8.

3. Almassi GH, Carr BM, Bishawi M, Shroyer AL, Quin JA, Hattler B, et al. Resident versus attending surgeon graft patency and clinical outcomes in on-versus off-pump coronary artery bypass surgery. J Thorac Cardiovasc Surg. 2015;150: 1428-37.e1. 